\title{
О НОРМАХ РАЗНОСТНЫХ ОПЕРАТОРОВ БОМАНА - ШАПИРО ${ }^{1}$
}

\section{А. Г. Бабенко, Ю. В. Крякин}

При заданных $k \in \mathbb{N}, h>0$ на пространстве $C=C(\mathbb{R})$ непрерывных ограниченных на вещественной оси $\mathbb{R}=(-\infty, \infty)$ функций рассматривается точное неравенство $\left\|W_{2 k}(f, h)\right\|_{C} \leq C_{k}\|f\|_{C}$ для разностного оператора Бомана-Шапиро вида $W_{2 k}(f, h)(x):=\frac{(-1)^{k}}{h} \int_{-h}^{h}\left(\begin{array}{c}2 k \\ k\end{array}\right)^{-1} \widehat{\Delta}_{t}^{2 k} f(x)\left(1-\frac{|t|}{h}\right) d t$, где $\widehat{\Delta}_{t}^{2 k} f(x):=\sum_{j=0}^{2 k}(-1)^{j}\left(\begin{array}{c}2 k \\ j\end{array}\right) f(x+j t-k t)$ - центральная конечная разность функции $f$ порядка $2 k$ с шагом $t$. При каждом фиксированном $k \in \mathbb{N}$ точная константа $C_{k}$ в указанном неравенстве является нормой оператора $W_{2 k}(\cdot, h)$ из $C$ в $C$. Доказано, что $C_{k}$ не зависит от $h$, возрастает по $k$ и предъявлен простой способ вычисления константы $C_{*}=\lim _{k \rightarrow \infty} C_{k}=2.6699263 \ldots$ с точностью $10^{-7}$. В работе также рассмотрена задача продолжения непрерывной функции $f$ с отрезка $[-1,1]$ на ось $\mathbb{R}$. Для этого продолжения $g_{f}:=g_{f, k, h}, k \in \mathbb{N}, 0<h<1 /(2 k)$, функций $f \in C[-1,1]$ получены новые двусторонние оценки для точной константы $C_{k}^{*}$ в неравенстве $\left\|W_{2 k}\left(g_{f}, h\right)\right\|_{C(\mathbb{R})} \leq C_{k}^{*} \omega_{2 k}(f, h)$, где $\omega_{2 k}(f, h)$ - модуль непрерывности функции $f$ порядка $2 k$. А именно, при любом натуральном $k \geq 6$ и любом $h \in(0,1 /(2 k))$ доказано двойное неравенство $5 / 12 \leq C_{k}^{*}<\left(2+e^{-2}\right) C_{*}$.

Ключевые слова: разностный оператор, $k$-ый модуль непрерывности, оценка нормы.

\section{A. G. Babenko, Yu. V. Kryakin. On the norms of Boman-Shapiro difference operators.}

For given $k \in \mathbb{N}$ and $h>0$, an exact inequality $\left\|W_{2 k}(f, h)\right\|_{C} \leq C_{k}\|f\|_{C}$ is considered on the space $C=C(\mathbb{R})$ of continuous functions bounded on the real axis $\mathbb{R}=(-\infty, \infty)$ for the Boman-Shapiro difference operator $W_{2 k}(f, h)(x):=\frac{(-1)^{k}}{h} \int_{-h}^{h}\left(\begin{array}{c}2 k \\ k\end{array}\right) \widehat{\Delta}_{t}^{2 k} f(x)\left(1-\frac{|t|}{h}\right) d t$, where $\widehat{\Delta}_{t}^{2 k} f(x):=\sum_{j=0}^{2 k}(-1)^{j}\left(\begin{array}{c}2 k \\ j\end{array}\right) f(x+j t-k t)$ is the central finite difference of a function $f$ of order $2 k$ with step $t$. For each fixed $k \in \mathbb{N}$, the exact constant $C_{k}$ in the above inequality is the norm of the operator $W_{2 k}(\cdot, h)$ from $C$ to $C$. It is proved that $C_{k}$ is independent of $h$ and increases in $k$. A simple method is proposed for the calculation of the constant $C_{*}=\lim _{k \rightarrow \infty} C_{k}=2.6699263 \ldots$ with accuracy $10^{-7}$. We also consider the problem of extending a continuous function $f$ from the interval $[-1,1]$ to the axis $\mathbb{R}$. For extensions $g_{f}:=g_{f, k, h}, k \in \mathbb{N}, 0<h<1 /(2 k)$, of functions $f \in C[-1,1]$, we obtain new twosided estimates for the exact constant $C_{k}^{*}$ in the inequality $\left\|W_{2 k}\left(g_{f}, h\right)\right\|_{C(\mathbb{R})} \leq C_{k}^{*} \omega_{2 k}(f, h)$, where $\omega_{2 k}(f, h)$ is the modulus of continuity of $f$ of order $2 k$. Specifically, for any natural $k \geq 6$ and any $h \in(0,1 /(2 k))$, we prove the double inequality $5 / 12 \leq C_{k}^{*}<\left(2+e^{-2}\right) C_{*}$.

Keywords: difference operator, $k$ th modulus of continuity, norm estimate.

MSC: $41 \mathrm{~A} 10,41 \mathrm{~A} 17,41 \mathrm{~A} 44$

DOI: $10.21538 / 0134-4889-2020-26-4-64-75$

\section{1. Введение}

Пусть $C:=C(\mathbb{R})$ - пространство непрерывных вещественнозначных ограниченных на $\mathbb{R}=(-\infty,+\infty)$ функций с обычной нормой $\|f\|_{C}=\sup \{|f(x)|: x \in \mathbb{R}\}$,

$$
\widehat{\Delta}_{t}^{2 k} f(x):=\sum_{j=0}^{2 k}(-1)^{j}\left(\begin{array}{c}
2 k \\
j
\end{array}\right) f(x+j t-k t)
$$

\footnotetext{
${ }^{1}$ Исследования поддержаны РФФИ (проект № 18-01-00336) и Программой повышения конкурентоспособности УрФУ (постановление № 211 Правительства РФ от 16.03.2013, контракт № 02.А03.21.0006 от 27.08.2013).
} 
- конечная центральная разность функции $f$ порядка $2 k$ с шагом $t \in \mathbb{R}$. В статье рассматривается разностный оператор $W_{2 k}(f, h), h>0$, действующий на функции $f \in C$ по правилу

$$
W_{2 k}(f, h)(x):=\frac{(-1)^{k}}{\left(\begin{array}{c}
2 k \\
k
\end{array}\right) h} \int_{-h}^{h} \widehat{\Delta}_{t}^{2 k} f(x)\left(1-\frac{|t|}{h}\right) d t .
$$

Этот оператор является частным случаем разностного оператора, введенного в 1967 г. Г. Шапиро [1] (см. также [2, с. 60; 3]). Он возник в наших исследованиях [4-6] в задачах вычисления точных констант в классических неравенствах Джексона - Стечкина между наилучшим равномерным приближением непрерывных вещественнозначных функций тригонометрическими полиномами $\tau_{n-1}(t)=\sum_{j=1-n}^{n-1} d_{j} e^{i j t}, d_{0} \in \mathbb{R}, d_{-j}=\bar{d}_{j} \in \mathbb{C}, j=1,2, \ldots, n-1$, на периоде (одномерном торе) $\mathbb{T}=\mathbb{R} /(2 \pi \mathbb{Z})$ (см. [7]) и алгебраическими многочленами $p_{n-1}(x)=\sum_{j=0}^{n-1} a_{j} x^{j}$, $a_{j} \in \mathbb{R}, j=0,1, \ldots, n-1$, на отрезке $\mathbb{I}=[-1,1]$ (см. [8]) и модулями непрерывности порядка $m$ для периодических и непериодических функций, определенных соответственно формулами

$$
\begin{gathered}
\widetilde{\omega}_{m}(f, \delta):=\sup _{x \in \mathbb{T}, 0<h \leq \delta}\left|\widehat{\Delta}_{h}^{m} f(x)\right|, \\
\omega_{m}(f, \delta):= \begin{cases}\sup _{x \in(1-m h / 2) \amalg, 0<h \leq \delta}\left|\widehat{\Delta}_{h}^{m} f(x)\right| & \text { при } 0<\delta \leq \frac{2}{m}, \\
\omega_{k}\left(f, \frac{2}{m}\right) & \text { при } \quad \delta>\frac{2}{m},\end{cases}
\end{gathered}
$$

где $\lambda \mathbb{I}:=\{\lambda t: t \in \mathbb{I}\}$. Сами неравенства Джексона - Стечкина имеют вид

$$
\begin{aligned}
& \widetilde{E}_{n}(f):=\inf _{\tau_{n-1}} \sup _{t \in \mathbb{T}}\left|f(t)-\tau_{n-1}(t)\right| \leq \widetilde{J}_{n}(m, \alpha) \widetilde{\omega}_{m}(f, \alpha \pi / n), \quad f \in C(\mathbb{T}) ; \\
& E_{n}(f):=\inf _{p_{n-1}} \sup _{x \in \mathbb{I}}\left|f(x)-p_{n-1}(x)\right| \leq J_{n}(m, \alpha) \omega_{m}(f, \alpha \pi / n), \quad f \in C(\mathbb{I}) ;
\end{aligned}
$$

здесь

$$
\begin{gathered}
\widetilde{J}_{n}(m, \alpha):=\sup _{f \in C(\mathbb{T}), f \neq \text { const }} \frac{\widetilde{E}_{n}(f)}{\widetilde{\omega}_{m}(f, \alpha \pi / n)}, \quad n, m \in \mathbb{N} ; \\
J_{n}(m, \alpha):=\sup _{f \in C(\mathbb{I}), f \notin \mathcal{P}_{m-1}} \frac{E_{n}(f)}{\omega_{m}(f, \alpha \pi / n)}, \quad n, m \in \mathbb{N}, \quad n \geq m ;
\end{gathered}
$$

$\alpha>0 ; \mathcal{P}_{m-1}-$ множество алгебраических полиномов степени не выше $m-1$ с вещественными коэффициентами.

В случае приближения периодических функций $f \in C(\mathbb{T})$ тригонометрическими полиномами справедливы оценки $[4 ; 5]$

$$
\left(\begin{array}{c}
2 k \\
k
\end{array}\right)^{-1} \leq \widetilde{J}_{n}(2 k, \alpha)<\left(\sec \frac{\pi}{2 \alpha}\right)\left(\begin{array}{c}
2 k \\
k
\end{array}\right)^{-1} \quad \text { при } \quad \alpha>1, \quad n, k \in \mathbb{N},
$$

а в случае приближения $f \in C(\mathbb{I})$ алгебраическими многочленами при любом натуральном $k \geq 5$ имеют место неравенства [6, теорема 1]

$$
\frac{1}{2} \leq J_{n}(2 k, \alpha)<3\left(2+e^{-2}\right)\left(2 \sec \frac{\pi}{2 \alpha}-1-\frac{\pi^{2}}{8 \alpha^{2}}\right) \quad \text { при } \quad 1<\alpha \leq \frac{2 k-1}{\pi}, \quad n \geq 2 k(2 k-1) .
$$

Отметим, что при $k=1,2,3,4$ имеют место более точные оценки сверху [6, теорема 2] для $J_{n}(2 k, \alpha)$, чем в $(1.6)$. 
В работе [5] (см. в ней доказательство леммы 1) было получено неравенство

$$
\left\|W_{2 k}(f, h)\right\|_{C} \leq 3\|f\|_{C}
$$

для любых $f \in C=C(\mathbb{R}), k \in \mathbb{N}$ и $h>0$.

В данной работе установлены два новых результата. Первый из них касается точной (наименьшей) константы $C_{k}$ в неравенстве

$$
\left\|W_{2 k}(f, h)\right\|_{C} \leq C_{k}\|f\|_{C}, \quad f \in C \quad(k \in \mathbb{N}, h>0),
$$

т. е. нормы оператора $W_{2 k}(\cdot, h): C \rightarrow C$

$$
C_{k}=\sup _{f \in C, f \neq 0} \frac{\left\|W_{2 k}(f, h)\right\|_{C}}{\|f\|_{C}} .
$$

Мы доказываем, что константы $C_{k}(k \in \mathbb{N})$ не зависят от $h$ и возрастают по $k$. Кроме того, в работе вычислено предельное значения $C_{*}=\lim _{k \rightarrow \infty} C_{k}=2.6699263 \ldots$ с точностью $10^{-7}$.

Наш второй результат относится к продолжению непрерывных функций с отрезка на ось с сохранением гладкости. Существенный вклад в эту тематику внес Х. Уитни [9-12]. Наша задача связана с исследованиями В. К. Дзядыка [13] и О. В. Бесова [14;15]. Мы рассматриваем продолжение $g_{f}:=g_{f, k, h}$ непрерывной функции $f$ с отрезка $\mathbb{I}$ на ось $\mathbb{R}$, предложенное в $[16$, лемма 2.3]. Здесь мы улучшаем оценку сверху для константы в неравенстве (2.4) из [6] между нормой функции $W_{2 k}\left(g_{f}, h\right)$ в $C(\mathbb{R})$ и модулем непрерывности функции $f \in C(\mathbb{I})$ порядка $2 k$ в точке $h$ при $0<h<(2 k)^{-1}$. Кроме того, показываем, что указанная константа не только ограничена по $k[6$, предложение 1$]$, но и отделена от нуля (см. теорему 2 ниже).

В дальнейшем через $i, j, k, l, m, n$ будем обозначать целые числа, а через $C(A)$ - пространство непрерывных функций на $A$ с нормой $\|f\|:=\|f\|_{C(A)}:=\sup _{x \in A}|f(x)|$; здесь $A-$ отрезок $\mathbb{I}=[-1,1]$ или ось $\mathbb{R}$.

Кроме классических модулей непрерывности порядка $m$ для периодических и непериодических функций (см. формулы (1.2), (1.3)) в прямых теоремах теории приближения функций важен оператор $W_{2 k}(\cdot, h)$ (см. (1.1)), который можно рассматривать как специальный модуль непрерывности Бомана-Шапиро [3]. Известно (см. $[4 ; 5 ; 17])$, что функция $W_{2 k}(f, h)(\cdot)$ представляет собой разность между функцией $f$ и некоторой линейной комбинацией вторых средних Стеклова функции $f$. Напомним обозначения свертки функций

$$
(f * g)(x):=\int_{\mathbb{R}} f(t) g(x-t) d t
$$

(здесь нам достаточно ограничиться случаем, когда $f \in L(\mathbb{R})$, а $g \in C(\mathbb{R})$ ), а также

$$
\chi_{h}^{2}(x):=\left(\chi_{h} * \chi_{h}\right)(x)= \begin{cases}\frac{1}{h}\left(1-\frac{|x|}{h}\right), & x \in[-h, h], \\ 0, & x \notin[-h, h],\end{cases}
$$

- сверточного квадрата нормализованной в $L$ характеристической функции $\chi_{h}$ отрезка $[-h / 2, h / 2]$.

При заданных $k \in \mathbb{N}, h>0$ формулой (1.1) определятся оператор Бомана-Шапиро не только для непрерывных на $\mathbb{R}$ функций, но и для локально интегрируемых функций $f: \mathbb{R} \rightarrow \mathbb{R}$, при этом имеют место следующие представления (см. [4;5]):

$$
W_{2 k}(f, h)(x)=\frac{(-1)^{k}}{\left(\begin{array}{c}
2 k \\
k
\end{array}\right)} \int_{\mathbb{R}} \widehat{\Delta}_{t}^{2 k} f(x) \chi_{h}^{2}(t) d t=f(x)-\left(\Lambda_{2 k} * f\right)(x), \quad x \in \mathbb{R},
$$

где

$$
\Lambda_{2 k, h}(x)=2 \sum_{j=1}^{k}(-1)^{j+1} a_{j, k} \chi_{j h}^{2}(x), \quad a_{j, k}:=\left(\begin{array}{c}
2 k \\
k+j
\end{array}\right) /\left(\begin{array}{c}
2 k \\
k
\end{array}\right) .
$$




\section{2. Формулировки результатов}

В дальнейшем будем использовать стандартное обозначение $\|g\|_{L}=\int_{\mathbb{R}}|g(u)| d u$ интегральной нормы абсолютно интегрируемой на $\mathbb{R}$ функции $g$. Из (1.11) следует неравенство

$$
\left|W_{2 k}(f, h)(x)\right| \leq\left(1+\left\|\Lambda_{2 k, h}\right\|_{L}\right) \sup _{t \in[x-k h, x+k h]}|f(t)|, \quad f \in C, \quad k \in \mathbb{N}, \quad h>0, \quad x \in \mathbb{R} .
$$

Ранее авторами (см. доказательство леммы 1 из [5]) было установлено, что при любых $k \in \mathbb{N}$ и $h>0$ выполняется неравенство

$$
\left\|\Lambda_{2 k, h}\right\|_{L}<2 .
$$

Для заданных $k \in \mathbb{N}, h>0$ обозначим через $C_{k}(x)$ точную константу в неравенстве

$$
\left|W_{2 k}(f, h)(x)\right| \leq C_{k}(x) \sup _{t \in[x-k h, x+k h]}|f(t)|, \quad f \in C,
$$

при фиксированном $x \in \mathbb{R}$. В силу инвариантности пространства $C$ и нормы $\|\cdot\|_{C}$ относительно любого сдвига величина $C_{k}(x)$ не зависит от $x$ и совпадает с $\left\|W_{2 k}(\cdot, h)\right\|_{C \rightarrow C}$, т. е. с величиной $C_{k}$, определенной формулой (1.8) и поэтому при любом $x \in \mathbb{R}$ имеют место равенства

$$
C_{k}(x)=1+\left\|\Lambda_{2 k, h}\right\|_{L}=1+\int_{\mathbb{R}}\left|\Lambda_{2 k, h}(t)\right| d t=C_{k} \quad(k \in \mathbb{N}, h>0) .
$$

Поэтому из неравенства (2.2) получаем оценку

$$
C_{k}<3, \quad k \in \mathbb{N}
$$

Для малых $k$ прямым вычислением нетрудно проверить, что [5, Remark 1]

$C_{1}=2, C_{2}=98 / 45, C_{3} \approx 2.26, C_{4} \approx 2.31, \ldots, C_{10} \approx 2.42, \ldots, C_{100} \approx 2.58 \ldots, C_{500} \approx 2.63$.

Здесь мы улучшаем оценку (2.5) при каждом $k \in \mathbb{N}$ и доказываем следующее утверждение.

Теорема 1. Константа $C_{k}$, определенная равенством (1.8), не зависит от $h$, возрастает nо $k$, причем

$$
C_{*}:=\lim _{k \rightarrow \infty} C_{k}=2.6699263 \ldots .
$$

Для формулировки второго утверждения нам понадобятся следующие обозначения. Пусть $k \in \mathbb{N}, 0<h<(2 k)^{-1}, f \in C(\mathbb{I}) ; p_{2 k-1}^{-}$и $p_{2 k-1}^{+}$- алгебраические полиномы (степени не выше $2 k-1)$ наилучшего равномерного приближения функции $f$ на отрезках $\mathbb{I}^{-}=[-1,-1+2 k h]$ и $\mathbb{I}^{+}=[1-2 k h, 1]$ соответственно. Следуя [16, лемма 2.3], положим

$$
g_{f}(x):= \begin{cases}p_{2 k-1}^{+}(x), & x \in(1,+\infty) \\ f(x), & x \in \mathbb{I} \\ p_{2 k-1}^{-}(x), & x \in(-\infty,-1)\end{cases}
$$

Для указанных $k$ и $h$ обозначим через $C_{k}^{*}=C_{k}^{*}(h)$ точную константу в неравенстве

$$
\left\|W_{2 k}\left(g_{f}, h\right)\right\|_{C(\mathbb{R})} \leq C_{k}^{*} \omega_{2 k}(f, h), \quad f \in C(\mathbb{I}) .
$$

C помощью теоремы 1 и методов, развитых нами в работе [6], получен следующий результат.

Теорема 2. Пусть $k \in \mathbb{N}, k \geq 6,0<h<(2 k)^{-1}$. Тогда для точной константы $C_{k}^{*}$ в неравенстве (2.8) имеют место оценки

$$
\frac{5}{12} \leq C_{k}^{*} \leq\left(2+e^{-2}\right) C_{k}<\left(2+e^{-2}\right) C_{*} .
$$




\section{3. Доказательства теорем}

Д о к а з а т е л ь с т в о теоремы 1 . Зафиксируем произвольные $k \in \mathbb{N}$ и $h>0$. В силу (2.4) задача нахождения величины $C_{k}$ сводится к вычислению интегральной нормы $\left\|\Lambda_{2 k, h}\right\|_{L}$ ядра $\Lambda_{2 k, h}$ на прямой $\mathbb{R}$. Для доказательства независимости от $h$ величины $C_{k}$ достаточно показать, что норма $\left\|\Lambda_{2 k, h}\right\|_{L}$ не зависит от $h$. Сопоставим ядру $\Lambda_{2 k, h}$ оператор $V_{2 k}(f, h)$, действующий и $C$ в $C$ по правилу

$$
V_{2 k}(f, h)(x)=\left(\Lambda_{2 k} * f\right)(x), \quad x \in \mathbb{R} .
$$

Норма $\left\|V_{2 k}(\cdot, h)\right\|_{C \rightarrow C}$ равна $\left\|\Lambda_{2 k, h}\right\|_{L}$ и совпадает с нормой следующего линейного функционала на пространстве $C: V_{2 k, h}(f):=V_{2 k}(f, h)(0)=\left(\Lambda_{2 k} * f\right)(0)$. Поэтому достаточно показать, что норма линейного функционала $V_{2 k, h}: C \rightarrow \mathbb{R}$ не зависит от $h$. Для этого применим следующее свойство треугольной функции (см. (1.10)):

$$
\chi_{j h}^{2}(x)=\frac{1}{h} \chi_{j}^{2}\left(\frac{x}{h}\right) \text { при любых } x \in \mathbb{R}, j>0, h>0 .
$$

Для произвольной функции $f \in C$ с помощью этого свойства, определения (1.12) ядра $\Lambda_{2 k, h}$, определения (1.9) операции свертки, а также известного свойства коммутативности этой операции и четности ядра $\Lambda_{2 k, h}$ получаем

$$
\begin{gathered}
\left(\Lambda_{2 k, h} * f\right)(0)=\int_{\mathbb{R}} f(t)\left(2 \sum_{j=1}^{k}(-1)^{j+1} a_{j, k} \chi_{j h}^{2}(t)\right) d t=\frac{1}{h} \int_{\mathbb{R}} f(t)\left(2 \sum_{j=1}^{k}(-1)^{j+1} a_{j, k} \chi_{j}^{2}\left(\frac{t}{h}\right)\right) d t \\
=\int_{\mathbb{R}} f(h u) \Lambda_{2 k, 1}(u) d u=\int_{\mathbb{R}} g(u) \Lambda_{2 k, 1}(u) d u=\left(\Lambda_{2 k, 1} * g\right)(0), \quad \text { где } \quad g(u)=f(h u) .
\end{gathered}
$$

Отсюда приходим к выводу, что нормы линейных функционалов $V_{2 k, h}, V_{2 k, 1}$ совпадают, поскольку пространство $C$ и норма в этом пространстве инвариантны относительно сжатия и растяжения аргумента функции. Таким образом, $\left\|\Lambda_{2 k, h}\right\|_{L}=\left\|\Lambda_{2 k, 1}\right\|_{L}$ при любых $k \in \mathbb{N}, h>0$. Поэтому далее будем рассматривать лишь случай $h=1$.

Ядро $\Lambda_{2 k}:=\Lambda_{2 k, 1}$ записывается следующим образом (см. доказательство леммы 2 из [6]):

$$
\Lambda_{2 k}(x)=2 \sum_{j=1}^{k}(-1)^{j+1} a_{j, k} \chi_{j}^{2}(x)=2 \sum_{j=-k+1}^{k-1} b_{j, k} \chi_{1}^{2}(x-j),
$$

с коэффициентами $b_{i, k}=b_{-i, k}, \quad b_{k, k}=b_{-k, k}=0$ и

$$
b_{i, k}=\sum_{j=i+1}^{k}(-1)^{j+1} \frac{(j-i) a_{j, k}}{j^{2}}, \quad i \geq 0, \quad a_{j, k}=\left(\begin{array}{c}
2 k \\
k+j
\end{array}\right) /\left(\begin{array}{c}
2 k \\
k
\end{array}\right) .
$$

Сумму, у которой нижний индекс суммирования больше верхнего, считаем равной нулю.

В [5, Lemma 1, Lemma A] было доказано, что

$$
(-1)^{i} b_{i, k}>0, \quad\left|b_{i, k}\right|>\left|b_{i+1, k}\right|, \quad i=0, \ldots, k-1 .
$$

Отсюда вытекает, что ядро $\Lambda_{2 k}$ является четной, непрерывной, кусочно-линейной функцией (см. [5, Fig. 1]) с носителем $[-k, k]$, при этом на каждом отрезке вида $[i, i+1](i=-k \ldots k-1)$ это ядро представляет собой линейную функцию. Таким образом, график ядра $\Lambda_{2 k}$ на $[-k, k]$ состоит из $2 k$ линейных звеньев с вершинами (точками излома) $\left(i, 2 b_{i, k}\right), i=-k \ldots k$. Справедливы также следующие неравенства (см. [5, Lemma 1, Lemma A]): $\left|b_{i, k}\right| \leq\left|b_{i, k+1}\right|, i=-k \ldots k$. 


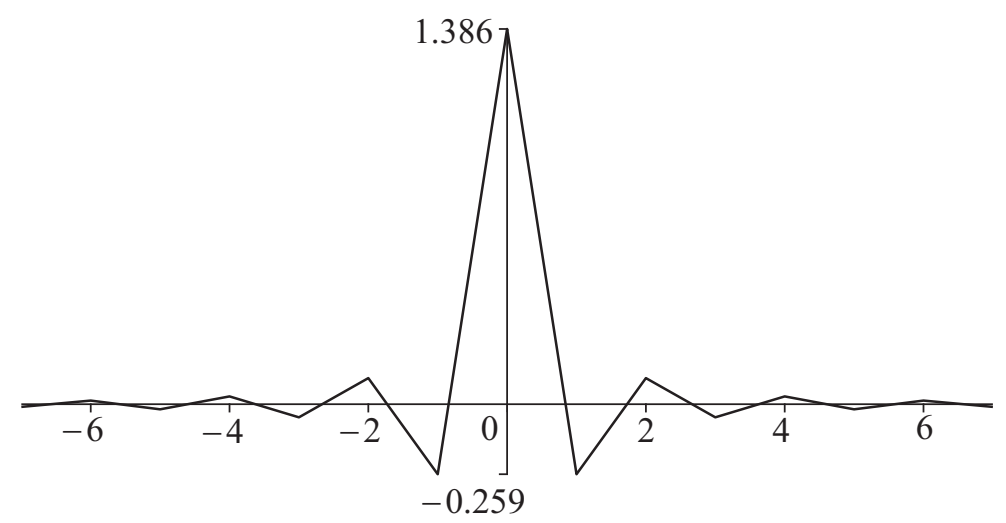

Ядро $\Lambda$

В предельном случае $(k \rightarrow \infty)$ получаем кусочно-линейную функцию $\Lambda=\Lambda_{\infty}$ (см. рисунок) с носителем $\mathbb{R}$ и вершинами (точками излома) $\left(i, 2 b_{i}\right)$, где

$$
2 b_{i}:=2 b_{i, \infty}:=2 \sum_{j=|i|+1}^{\infty}(-1)^{j+1} \frac{1}{j}\left(1-\frac{|i|}{j}\right) .
$$

В дальнейшем будем использовать обозначения

$$
c_{j, k}:=(-1)^{j} b_{j, k}, \quad c_{j}:=c_{j, \infty}=(-1)^{j} b_{j} .
$$

Можно считать, что $c_{j, k}=0$ для $|j| \geq k$. В [5, Lemma A] было доказано, что $c_{j, k} \geq 0, c_{j} \geq 0$ и $c_{j+1, k} \leq c_{j, k}, c_{j+1} \leq c_{j}$.

Вычислим норму функции $\Lambda$ в пространстве $L$. Имеем

$$
\int_{\mathbb{R}}|\Lambda(t)| d t=\sum_{j=-\infty}^{\infty} \int_{j}^{j+1}|\Lambda(t)| d t=2 \sum_{j=0}^{\infty} \int_{j}^{j+1}|\Lambda(t)| d t,
$$

положительные нули ядра $\Lambda(x)$ имеют вид: $x_{i}=i-1+\frac{c_{i-1}}{c_{i-1}+c_{i}}, i=1,2, \ldots$ Следовательно,

$$
\begin{aligned}
& \frac{1}{2} \int_{\mathbb{R}}|\Lambda(t)| d t=\sum_{j=0}^{\infty} \int_{j}^{j+1}|\Lambda(t)| d t=c_{0} x_{1}+c_{1}\left(1-x_{1}\right)+c_{1}\left(x_{2}-1\right)+c_{2}\left(2-x_{2}\right)+c_{2}\left(x_{3}-2\right)+\ldots \\
& \quad=c_{0} \frac{c_{0}}{c_{0}+c_{1}}+c_{1}\left(1-\frac{c_{0}}{c_{0}+c_{1}}\right)+c_{1} \frac{c_{1}}{c_{1}+c_{2}}+c_{2}\left(1-\frac{c_{1}}{c_{1}+c_{2}}\right)+\cdots=\sum_{j=0}^{\infty} \frac{c_{j}^{2}+c_{j+1}^{2}}{c_{j}+c_{j+1}} .
\end{aligned}
$$

Лемма. При любых $k \in \mathbb{N}$ имеем

$$
\int_{\mathbb{R}}\left|\Lambda_{2 k}(t)\right| d t<\int_{\mathbb{R}}\left|\Lambda_{2 k+2}(t)\right| d t<\int_{\mathbb{R}}|\Lambda(t)| d t=2 \sum_{j=0}^{\infty} \frac{c_{j}^{2}+c_{j+1}^{2}}{c_{j}+c_{j+1}} .
$$

Д о к а з а т е л ь с т в о. Последнее равенство в (3.5) равносильно (3.4). Неравенства в (3.5) следуют из того (см. доказательство леммы А в [5]), что $c_{i, k+1}-c_{i, k}>c_{i+1, k+1}-c_{i+1, k}>0$, $i=0, \ldots, k-1$. Действительно, в обозначениях $c_{i, k}=u, c_{i+1, k}=v, c_{i, k+1}=x, c_{i+1, k+1}=y$ достаточно показать, что

$$
\frac{x^{2}+y^{2}}{x+y}>\frac{u^{2}+v^{2}}{u+v}
$$


при условиях $x>y>0, u>v>0, x-u>y-v>0$. Неравенство (3.6) эквивалентно неравенству

$$
x u(x-u)+x v(x-v)+y v(y-v)+y u(y-u)>0 .
$$

Предположим, что $v<y<u<x$, так как если $y>u$, то доказывать нечего. Положим $y-v=t$ и заменим $x-u$ на $t$. Получим, после простых выкладок, что левая часть (3.7) больше

$$
2 t(y u+(u+t)(y-t))=2(y-v)(y u+x v)>0 .
$$

Для завершения доказательства теоремы 1 осталось вычислить норму в $L$ функции $\Lambda$ с точностью $10^{-7}$, т. е. установить, что

$$
\int_{\mathbb{R}}|\Lambda(t)| d t=1.6699263 \ldots
$$

Введем обозначения $\phi_{i}:=\sum_{j=i+1}^{\infty} \frac{(-1)^{i+j+1}}{j^{2}}, \psi_{i}:=\sum_{j=i+1}^{\infty} \frac{(-1)^{j+i+1}}{j}, i=0,1,2,3, \ldots$

Имеем

$$
\begin{gathered}
c_{i}=\psi_{i}-i \phi_{i}, \quad c_{i}+c_{i+1}=\phi_{i}, \\
c_{i}-c_{i+1}=c_{i}-\left(\phi_{i}-c_{i}\right)=2 \psi_{i}-(2 i+1) \phi_{i}>0 .
\end{gathered}
$$

Эти тождества легко проверяются и следуют из определения $c_{j}$. Неравенство $c_{i}>c_{i+1}$ и равенство $c_{i}+c_{i+1}=\phi_{i}$ доказаны в [5, Lemma A].

Воспользуемся тождествами (3.9), (3.10) и вычислим

$$
\sum_{j=0}^{\infty} \frac{c_{j}^{2}+c_{j+1}^{2}}{c_{j}+c_{j+1}}=\sum_{j=0}^{\infty} c_{j}-\sum_{j=0}^{\infty} c_{j+1} \frac{c_{j}-c_{j+1}}{c_{j}+c_{j+1}} .
$$

Несложно показать, что

$$
\sum_{j=0}^{\infty} c_{j}=\frac{1}{2}\left(\ln (2)+\frac{\pi^{2}}{8}\right)
$$

Действительно, это равенство вытекает из следующего тождества для коэффициентов:

$$
b_{i, k}=\sum_{j=|i|+1}^{k}(-1)^{j+1} \frac{(j-|i|) a_{j, k}}{j^{2}}
$$

а именно (см. [6])

$$
\sum_{j=-k+1}^{k-1}\left|b_{j, k}\right|=\sum_{j=-k+1}^{k-1}(-1)^{j} b_{j, k}=\sum_{i=1, i \text { odd }}^{k} \frac{a_{i, k}}{i^{2}} .
$$

В самом деле, последнее тождество влечет

$$
\sum_{j=-\infty}^{\infty} c_{j}=\sum_{j=-\infty}^{\infty}\left|b_{j, \infty}\right|=\sum_{i=1}^{\infty} \frac{1}{(2 i-1)^{2}}=\frac{\pi^{2}}{8} .
$$

Поэтому

$$
2 \sum_{j=1}^{\infty} c_{j}=\sum_{j=-\infty}^{\infty} c_{j}-c_{0}=\frac{\pi^{2}}{8}-\ln (2)
$$

и

$$
\sum_{j=0}^{\infty} c_{j}=c_{0}+\sum_{j=1}^{\infty} c_{j}=\ln (2)+\frac{1}{2}\left(\frac{\pi^{2}}{8}-\ln (2)\right)=\frac{1}{2}\left(\frac{\pi^{2}}{8}+\ln (2)\right) .
$$


Используя (3.12) мы можем подсчитать сумму ряда

$$
\sum_{j=0}^{\infty} c_{j+1} \frac{c_{j}-c_{j+1}}{c_{j}+c_{j+1}}
$$

с любой степенью точности, так как $0<\frac{c_{j}-c_{j+1}}{c_{j}+c_{j+1}}<1$ и (см. [5, с. 359]) $c_{j}<c_{j}+c_{j+1}<1 /(1+j)^{2}$. Некоторые вычисления, связанные с оценками рядов (3.11), приведены в приложении (разд. 4). Они приводят к равенству (3.8).

Теорема доказана.

Прежде чем перейти к доказательству теоремы 2, напомним современный вариант неравенства (теоремы) Уитни между наилучшим равномерным приближением непрерывной на конечном отрезке $[a, b]$ функции алгебраическими полиномами степени не выше $m-1$ через $m$-й модуль непрерывности функции. А именно, справедлив следующий вариант теоремы Уитни [20, Theorem 4.1] с наименьшей на настоящий момент константой в случае больших порядков $m$ модулей непрерывности $(m \geq 82000)$.

Теорема А. Пусть $f \in C[a, b],-\infty<a<b<\infty, m \in \mathbb{N}$. Тогдa

$$
E_{m-1}(f)_{C[a, b]} \leq\left(2+e^{-2}\right) \omega_{m}\left(f, \frac{b-a}{m}\right) .
$$

Д о к а з а т е л ь с т в о теоремы 2. Теорема А (при $m=2 k$ ) влечет неравенство

$$
\sup _{x \in \mathbb{I}^{ \pm}}\left|f(x)-p_{2 k-1}^{ \pm}(x)\right| \leq\left(2+e^{-2}\right) \omega_{2 k}(f, h) .
$$

Докажем, что $\left\|W_{2 k}\left(g_{f}, h\right)\right\|_{C(\mathbb{R})}=\left\|g_{f}-\Lambda_{2 k, h} * g_{f}\right\|_{C(\mathbb{R})} \leq\left(2+e^{-2}\right) C_{*} \omega_{2 k}(f, h)$. Из соображений симметрии следует, что достаточно рассмотреть случаи 1) $x \in[0,1-k h], 2) x \in(1-k h, 1]$, 3) $x \in(1,+\infty)$.

В первом случае по определению (1.11) имеем $\left|W_{2 k}\left(g_{f}, h\right)(x)\right| \leq\left(\begin{array}{c}2 k \\ k\end{array}\right)^{-1} \omega_{2 k}(f, h)$.

Во втором случае $x+k h \geq 1$. Тождество $W_{2 k}\left(p_{2 k-1}^{+}, h\right)(x) \equiv 0$ дает

$$
W_{2 k}\left(g_{f}, h\right)(x)=W_{2 k}\left(g_{f}-p_{2 k-1}^{+}, h\right)(x)+W_{2 k}\left(p_{2 k-1}^{+}, h\right)(x)=W_{2 k}\left(g_{f}-p_{2 k-1}^{+}, h\right)(x) .
$$

Отсюда с помощью теоремы 1

$$
\left|W_{2 k}\left(g_{f}-p_{2 k-1}^{+}, h\right)(x)\right| \leq C_{k} \sup _{t \in[x-k h, x+k h]}\left|g_{f}(t)-p_{2 k-1}^{+}(t)\right| \leq C_{*} \sup _{t \in[x-k h, x+k h]}\left|g_{f}(t)-p_{2 k-1}^{+}(t)\right|,
$$

и теоремы $\mathrm{A}$

$$
\sup _{x \in I^{+}} \mid f(x)-p_{2 k-1}^{+}(x) \leq\left(2+e^{-2}\right) \omega_{2 k}(f, h),
$$

выводим оценку во втором случае.

Третий случай аналогичен второму, когда $x \in(1,1+k h]$. Если же $x>1+k h$, то $2 k$-я разность равна нулю. Таким образом, доказаны оценки

$$
\begin{array}{ll}
\left\|W_{2 k}\left(g_{f}, h\right)\right\|_{C(\mathbb{R})} \leq C_{k}\left(2+e^{-2}\right) \omega_{2 k}(f, h), & 0<h<(2 k)^{-1}, \\
\left\|W_{2 k}\left(g_{f}, h\right)\right\|_{C(\mathbb{R})} \leq C_{*}\left(2+e^{-2}\right) \omega_{2 k}(f, h), & 0<h<(2 k)^{-1} .
\end{array}
$$

Эти оценки равносильны оценкам сверху константы $C_{k}^{*}$, содержащихся в $(2.9)$.

Вышеприведенные оценки и методы работы [6] позволяют уточнить оценку сверху в (1.6) для $J_{n}(2 k, \alpha)$, а именно, позволяют в указанной оценке поставить $C_{*}$ вместо 3 . Таким образом, при любом натуральном $k \geq 5$ выполняются неравенства

$$
\frac{1}{2} \leq J_{n}(2 k, \alpha) \leq U(\alpha) \quad \text { при } \quad 1<\alpha \leq \frac{2 k-1}{\pi}, \quad n \geq 2 k(2 k-1),
$$


где $U(\alpha)=C_{*}\left(2+e^{-2}\right)\left(2 \sec \frac{\pi}{2 \alpha}-1-\frac{\pi^{2}}{8 \alpha^{2}}\right)$. Заметим, что функция $U(\alpha)$ убывает при $\alpha>1$. Для $k \geq 6, h \in(0,1 /(2 k))$ выберем $n \geq 2 k(2 k-1), \alpha \geq 9 / \pi$, так, чтобы $h=\alpha \pi / n$. Теперь, если вместо множителя $C_{*}\left(2+e^{-2}\right)$ в правой части неравенства (3.14) написать множитель $(5 / 12-\delta)$, где $0<\delta<5 / 12$, то мы получим неравенства

$$
\frac{1}{2} \leq J_{n}(2 k, \alpha) \leq U(9 / \pi)<\left(\frac{5}{12}-\delta\right) \frac{6}{5} \quad \text { при } \quad n \geq 2 k(2 k-1), k \geq 6,
$$

которые являются противоречивыми. Отсюда следует оценка снизу константы $C_{k}^{*}$, содержащаяся в (2.9).

Теорема доказана.

3 а м е ч а н и е 1. Неравенства (3.15) являются усилением теоремы 1 из [6].

3 а м е ч а н и е 2. Напомним, что величина $2+e^{-2}$ является наилучшей известной оценкой константы в теореме Уитни (см. [18-20]). Отметим, что если справедлива гипотеза Сендова (константы Уитни ограничены сверху единицей), то множитель $\left(2+e^{-2}\right)$ в $(2.9)$ перед $C_{k}$ и перед $C_{*}$ можно убрать. Известно, что при малых $m \leq 8$ гипотеза Сендова верна.

\section{4. Приложение}

Здесь мы приведем дополнительные формулы и оценки для коэффициентов $c_{j}$ (см. (3.3)) и их сумм, необходимые для вычисления предельного значения $C_{*}$ нормы оператора $W_{2 k}$ при $k \rightarrow \infty$ с точностью $10^{-7}$. Сначала выпишем несколько первых коэффициентов $c_{j}$. Этих явных выражений достаточно для вычисления $C_{*}$ с точностью $10^{-7}$.

$$
\begin{array}{ll}
c_{0}=\ln 2=0.69314718 \ldots, & c_{1}=\frac{\pi^{2}}{12}-\ln 2=0.12931985 \ldots, \\
c_{2}=\ln 2-2 \cdot \frac{\pi^{2}}{12}+1=0.04821311 \ldots, & c_{3}=3 \cdot \frac{\pi^{2}}{12}-\ln 2-\frac{7}{4}=0.02425391 \ldots, \\
c_{4}=\ln 2-4 \cdot \frac{\pi^{2}}{12}+\frac{47}{18}=0.01439015 \ldots, & c_{5}=5 \cdot \frac{\pi^{2}}{1}-\ln 2-\frac{491}{144}=0.00946576 \ldots, \\
c_{6}=\ln 2-6 \cdot \frac{\pi^{2}}{12}+\frac{2549}{600}=0.00667831 \ldots, & c_{7}=7 \cdot \frac{\pi^{2}}{12}-\ln 2-\frac{61071}{1200}=0.00495538 \ldots, \\
c_{8}=\ln 2-8 \cdot \frac{\pi^{2}}{12}+\frac{28863}{4900}=0.00381907 \ldots, & c_{9}=9 \cdot \frac{\pi^{2}}{12}-\ln 2-\frac{1577257}{235200}=0.00303146 \ldots
\end{array}
$$

Запишем оценки величин

$$
\phi_{i}:=\sum_{j=i+1}^{\infty} \frac{(-1)^{i+j+1}}{j^{2}}, \quad \psi_{i}:=\sum_{j=i+1}^{\infty} \frac{(-1)^{j+i+1}}{j}, \quad i=0,1,2,3, \ldots,
$$

с помощью формулы Эйлера - Маклорена:

$$
\sum_{i=m+1}^{n} f(i)=\int_{m}^{n} f(x) d x+\frac{f(n)-f(m)}{2}+\sum_{k=1}^{\lfloor p / 2\rfloor} \frac{B_{2 k}}{(2 k) !}\left(f^{(2 k-1)}(n)-f^{(2 k-1)}(m)\right)+R,
$$

где $B_{2 k}=(-1)^{k+1} \frac{2 \zeta(2 k)(2 k) !}{(2 \pi)^{2 k}}-$ числа Бернулли, $\zeta(p)=\sum_{j=1}^{\infty} j^{-p}-$ дзета-функция Римана, $|R| \leq \frac{2 \zeta(p)}{(2 \pi)^{p}} \int_{m}^{n}\left|f^{(p)}(x)\right| d x$. 
Предположим, что $i \geq 10$. Для $\psi_{i}, \phi_{i}$ с точностью $i^{-9}$ имеем

$$
\begin{gathered}
\psi_{i} \approx \tilde{\psi}_{i}=1 /(2 i)-1 /\left(4 i^{2}\right)+1 /\left(8 i^{4}\right)-1 /\left(4 i^{6}\right)+17 /\left(16 i^{8}\right), \\
\phi_{2 i} \approx \tilde{\phi}_{2 i}=1 /\left(8 i^{2}\right)-1 /\left(16 i^{3}\right)+1 /\left(64 i^{5}\right)-3 /\left(256 i^{7}\right)+17 /\left(1024 i^{9}\right), \\
\phi_{2 i-1} \approx \tilde{\phi}_{2 i-1}=1 /\left(8 i^{2}\right)+1 /\left(16 i^{3}\right)-1 /\left(64 i^{5}\right)+3 /\left(256 i^{7}\right)-17 /\left(1024 i^{9}\right),
\end{gathered}
$$

и поэтому, с точностью, $i^{-8}$ получаем $c_{i}=\psi_{i}-i \phi_{i} \approx \tilde{c}_{i}=1 /\left(4 i^{2}\right)-3 /\left(8 i^{4}\right)+5 /\left(4 i^{6}\right)-119 /\left(16 i^{8}\right)$. Например, $\left|c_{10}-\tilde{c}_{10}\right|=7 \cdot 10^{-9}<10^{-8},\left|c_{20}-\tilde{c}_{20}\right|<7 \cdot 10^{-12}<10^{-11}<20^{-8}$.

Сейчас мы с помощью (3.12) можем вычислить приближенно величину $C_{*}$ (см. (2.6)) следующим образом:

$$
\begin{aligned}
C_{*}= & 1+\lim _{k \rightarrow \infty} \int_{\mathbb{R}}\left|\Lambda_{2 k}(t)\right| d t=1+\int_{\mathbb{R}}|\Lambda(t)| d t=1+2 \sum_{j=0}^{\infty} \frac{c_{j}^{2}+c_{j+1}^{2}}{c_{j}+c_{j+1}} \\
& =1+2\left(\sum_{j=0}^{\infty} c_{j}-\sum_{j=1}^{\infty} c_{j} \frac{c_{j-1}-c_{j}}{c_{j-1}+c_{j}}\right)=1+\ln 2+\frac{\pi^{2}}{8}-s,
\end{aligned}
$$

где

$$
s \approx 2\left(\sum_{j=1}^{9} c_{j} \frac{c_{j-1}-c_{j}}{c_{j-1}+c_{j}}+\sum_{j=10}^{\infty} \tilde{c}_{j} \frac{\tilde{c}_{j-1}-\tilde{c}_{j}}{\tilde{c}_{j-1}+\tilde{c}_{j}}\right)=0.25411037 \ldots+0.00281097 \ldots=0.25692135 \ldots
$$

Таким образом, имеем $C_{*}=1+\ln 2+\pi^{2} / 8-s=2.6699263 \ldots$.

Благодарность. Авторы искренне признательны В. Т. Шевалдину за ряд ценных замечаний, повлиявших на существенное улучшение изложения работы.

\section{СПИСОК ЛИТЕРАТУРЫ}

1. Shapiro H.S. A Tauberian theorem related to approximation theory // Acta Math. 1968. Vol. 120. P. 279-292.

2. Shapiro H.S. Smoothing and approximation of functions. New York etc.: Van Nostrand Reinhold Company, 1969. VIII, 134 p.

3. Boman J., Shapiro H.S. Comparison theorems for a generalized modulus of continuity // Ark. Mat. 1971. Vol. 9, no. 1-2. P. 91-116.

4. Foucart S., Kryakin Y., Shadrin A. On the exact constant in the Jackson-Stechkin inequality for the uniform metric // Constr. Approx. 2009. Vol. 29, no. 2. P. 157-179.

5. Babenko, A.G., Kryakin Yu.V., Staszak P.T. Special Moduli of Continuity and the Constant in the Jackson-Stechkin Theorem // Constr. Approx. 2013. Vol. 38, no 3. P. 339-364.

6. Бабенко А.Г., Крякин Ю.В. О константах в теореме Джексона-Стечкина в случае приближения алгебраическими многочленами // Тр. МИАН. 2018. Т. 303. С. 26-38.

7. Стечкин С.Б. О порядке наилучших приближений непрерывных функций // Изв. АН СССР. Сер. математическая. 1951. Т. 15, № 3. С. 219-242.

8. Брудный Ю.А. Приближение функций алгебраическими многочленами // Изв. АН СССР. Сер. математическая. Т. 32, № 4. С. 780-787.

9. Whitney H. Analytic extensions of differentiable functions defined in closed sets // Trans. Am. Math. Soc. 1934. Vol. 36. P. 63-89.

10. Whitney H. Functions differentiable on the boundaries of regions // Annals of Math. Second Series. 1934. Vol. 35. P. 482-485.

11. Whitney H. Differentiable functions defined in arbitrary subsets of Euclidean space // Trans. Amer. math. Soc. 1936. Vol. 40. P. 309-317.

12. Whitney H. On the extension of differentiable functions // Bull. Am. Math. Soc. 1944. Vol. 50. P. 76-81. 
13. Дзядык В.К. О продолжении функций, удовлетворяющих условию Липшица в метрике $L_{p} / /$ Мат. сб. 1956. Т. 40 (82), № 2. С. 239-242.

14. Бесов О.В. О продолжении функций с сохранением свойств интегрального модуля гладкости второго порядка // Мат. сб. 1962. Т. 58 (100), № 2. С. 673-684.

15. Бесов О.В. Продолжение функций за пределы области с сохранением дифференциальноразностных свойств в $L_{p} / /$ Мат. сб. 1965. Т. 66 (108), № 1. С. 80-96.

16. Виноградов О.Л., Жук В.В. Оценки функционалов с известным конечным набором моментов через модули непрерывности высоких порядков в пространствах функций, заданных на отрезке // Алгебра и анализ. 2013. Т. 25, № 3. С. 86-120.

17. Stekloff W. Sur les problemes de représentation des fonctions a l'aide de polynomes, du calcul approché des intégrales définies, du développement des fonctions en séries infinies suivant les polynomes et de l'interpolation, considérés au point de vue des idées de Tchebycheff. // Proc. Int. Math. Congr. (Toronto, 1924). Toronto: Univ. Toronto Press, 1928. Vol. 1. P. 631-640.

18. Whitney H. On the functions with bounded $n$-th differences // J. Math. Pures Appl., IX. Sér. 1957. Vol. 36. P. 67-95.

19. Kryakin Yu.V. Whitney's constants and Sendov's conjectures // Math. Balkanica, New Ser. 2002. Vol. 16, no. 1-4. P. 235-247.

20. Gilewicz J., Shevchuk I.A., Kryakin Yu.V. Boundedness by 3 of the Whitney interpolation constant // J. Approx. Theory. 2002. Vol. 119, no. 2. P. 271-290.

21. Kryakin Yu.V. On the theorem of H.Whitney in spaces $L_{p}, 1 \leq p \leq \infty / /$ Math. Balkanica, New Ser. 1990. Vol. 4, no. 3. P. 258-271.

22. Крякин, Ю.В., Коваленко, Л.Г. О константах Уитни в классах $L^{p}, 1 \leq p \leq \infty / /$ Изв. вузов. Матем. 1992. № 1. С. 69-77.

Поступила 13.07.2020

После доработки 15.11.2020

Принята к публикации 23.11.2020

Бабенко Александр Григорьевич

д-р физ.-мат. наук, зав. отд.

Институт математики и механики им. Н. Н. Красовского УрО РАН;

Уральский федеральный университет

г. Екатеринбург

e-mail: babenko@imm.uran.ru

Kryakin, Yuriy

dr hab.

Mathematical Institute

University of Wroclaw

e-mail: kryakin@math.uni.wroc.pl

\section{REFERENCES}

1. Shapiro H.S. A Tauberian theorem related to approximation theory. Acta Math., 1968, vol. 120, pp. 279-292. doi 10.1007/BF02394612 .

2. Shapiro H.S. Smoothing and approximation of functions. N Y etc.: Van Nostrand Reinhold Company, 1969, 134 p. ISBN: 044207526X.

3. Boman J., Shapiro H.S. Comparison theorems for a generalized modulus of continuity. Ark. Mat., 1971, vol. 9, no. 1-2, pp. 91-116. doi 10.1007/BF02383639.

4. Foucart S., Kryakin Y., Shadrin A. On the Exact Constant in the Jackson-Stechkin Inequality for the Uniform Metric. Constr. Approx., 2009, vol. 29, no. 2, pp. 157-179. doi 10.1007/s00365-008-9039-6 .

5. Babenko A.G., Kryakin Y.V., Staszak P.T. Special Moduli of Continuity and the Constant in the Jackson-Stechkin Theorem. Constr. Approx., 2013, vol. 38, no. 3, pp. 339-364.

doi 10.1007/s00365-013-9210-6.

6. Babenko A.G., Kryakin Yu.V. On constants in the Jackson-Stechkin theorem in the case of approximation by algebraic polynomials. Proc. Steklov Inst. Math., 2018, vol. 303, pp. 18-30.

doi 10.1134/S0081543818080035. 
7. Stechkin S.B. On the order of the best approximations of continuous functions. Izv. Akad. Nauk SSSR. Ser. Mat., 1951, vol. 15, no. 3, pp. 219-242 (in Russian).

8. Brudnii Yu.A. The approximation of functions by algebraic polynomials. Math. USSR-Izv., 1968, vol. 2, no. 4, pp. 735-743. doi 10.1070/IM1968v002n04ABEH000662 .

9. Whitney H. Analytic extensions of differentiable functions defined in closed sets. Trans. Am. Math. Soc., 1934, vol. 36, no. 1, pp. 63-89. doi 10.2307/1989708.

10. Whitney H. Functions differentiable on the boundaries of regions. Annals of Math. Second Series, 1934, vol. 35, no. 3, pp. 482-485. doi 10.1007/978-1-4612-2972-8_18.

11. Whitney H. Differentiable functions defined in arbitrary subsets of Euclidean space. Trans. Amer. math. Soc., 1936, vol. 40, no. 2, pp. 309-317. doi 10.1090/S0002-9947-1936-1501875-0 .

12. Whitney H. On the extension of differentiable functions. Bull. Am. Math. Soc., 1944, vol. 50, no. 2, pp. 76-81. doi 10.1090/S0002-9904-1944-08082-8.

13. Dzyadyk V.K. Continuation of functions satisfying a Lipschitz condition in the $L_{p}$ metric. Mat. Sb. (N.S.), 1956, vol. 40(82), no. 2, pp. 239-242 (in Russian).

14. Besov O.V. On the continuation of functions with preservation of the second-order integral modulus of smoothness. Mat. Sb. (N.S.), 1962, vol. 58(100), no. 2, pp. 673-684 (in Russian).

15. Besov O.V. Extension of functions beyond the boundary of a region with preservation of differentialdifference properties in $L_{p}$. Mat. Sb. (N.S.), 1965, vol. 66(108), no. 1, pp. 80-96 (in Russian).

16. Vinogradov O.L., Zhuk V.V. Estimates for functionals with a known finite set of moments in terms of high order moduli of continuity in spaces of functions defined on a segment. St. Petersburg Math. J., 2014, vol. 25, no. 3, pp. 421-446. doi: 10.1090/S1061-0022-2014-01297-6 .

17. Stekloff W. Sur les problemes de représentation des fonctions a l'aide de polynomes, du calcul approché des intégrales définies, du développement des fonctions en séries infinies suivant les polynomes et de l'interpolation, considérés au point de vue des idées de Tchebycheff. Proc. Int. Math. Congr., Toronto, 1924. Toronto: Univ. Toronto Press, 1928, vol. 1, pp. 631-640.

18. Whitney H. On the functions with bounded $n$-th differences. J. Math. Pures Appl., IX. Sér., 1957, vol. 36 , pp. $67-95$.

19. Kryakin Yu.V. Whitney's constants and Sendov's conjectures. Math. Balkanica, New Ser., 2002, vol. 16, no. $1-4$, pp. $235-247$.

20. Gilewicz J., Shevchuk I.A., Kryakin Yu.V. Boundedness by 3 of the Whitney Interpolation Constant. J. Approx. Theory, 2002, vol. 119, no. 2, pp. 271-290. doi: 10.1006/jath.2002.3732 .

21. Kryakin Yu.V. On the theorem of H.Whitney in spaces $L_{p}, 1 \leq p \leq \infty$. Math. Balkanica, New Ser., 1990, vol. 4, no 3, pp. 258-271.

22. Kryakin Yu.V., Kovalenko L.G. Whitney constants in the classes $L^{p}, 1 \leq p \leq \infty$. Russian Math. (Iz. VUZ), 1992, vol. 36 , no. 1 , pp. 68-75.

Received July 13, 2020

Revised November 15, 2020

Accepted November 23, 2020

Funding Agency: This work was supported by the Russian Foundation for Basic Research (project no. 18-01-00336) and by the Russian Academic Excellence Project (agreement no. 02.A03.21.0006 of August 27, 2013, between the Ministry of Education and Science of the Russian Federation and Ural Federal University), and as part of research conducted in the Ural Mathematical Center.

Aleksandr Grigor'evich Babenko, Dr. Phys.-Math. Sci., Krasovskii Institute of Mathematics and Mechanics of the Ural Branch of the Russian Academy of Sciences, Yekaterinburg, 620108 Russia; Ural Federal University, Yekaterinburg, 620000 Russia, e-mail: babenko@imm.uran.ru .

Yuriy Kryakin, dr hab., Mathematical Institute of University of Wroclaw, 48-300 Wroclaw, Poland e-mail: kryakin@math.uni.wroc.pl.

Cite this article as: A. G. Babenko, Yu. V. Kryakin. On the norms of Boman-Shapiro difference operators, Trudy Instituta Matematiki i Mekhaniki UrO RAN, 2020, vol. 26, no. 4, pp. 64-75. 